\title{
Minimally invasive liver surgery for metastases from colorectal cancer: oncologic outcome and prognostic factors
}

\author{
Baki Topal
}

Received: 15 October 2012/ Accepted: 13 November 2012/Published online: 10 January 2013

(C) Springer Science+Business Media New York 2013

I enjoyed reading the Letter to the Editor from professor Hottenrott, and I fully agree that novel molecular markers may lead us to better predict the oncologic outcome for patients with liver metastases from colorectal cancer. I also want to emphasize that surgery still remains the mainstay of treatment for these patients.

With respect to a sentence in professor Hottenrott's letter quoted later, I must comment that there may be a selection bias in our study because it was not randomized, although major hepatectomies also were performed via minimally invasive liver surgery (MILS). In major hepatectomies too, the oncologic outcome was comparable between MILS and open surgery. These data have been published in the recent issue of Surgical Endoscopy.
The sentence in question reads: "Perhaps, for larger liver metastases which presented difficulties for complete resection (R0), an open approach was performed by Topal and colleagues that has resulted in bias in favour of MILS group." This sentence is not correct. In my opinion, as outlined in our article, any future randomized controlled trial (RCT) on colorectal liver metastases (CRLM) needs to be stratified for many potential predictors of survival, and the opportunity to conduct a well-designed RCT evaluating the efficacy of MILS for CRLM seems to be very unlikely.

Disclosures Baki Topal has no conflicts of interest or financial ties to disclose.
B. Topal $(\bowtie)$

Department of Abdominal Surgery, University Hospitals Leuven KUL, Herestraat 49, 3000 Leuven, Belgium

e-mail: baki.topal@uzleuven.be 